\title{
O EMPREGO DA ENGENHARIA KANSEI NO DESENVOLVIMENTO DE MATERIAIS DE APRENDIZAGEM
}

\author{
Msc. Adriana Sugimoto \\ Universidade Federal do Rio Grande do Sul - UFRGS \\ adri.sugimoto@gmail.com \\ Dra. Tânia Luisa Koltermann da Silva \\ Universidade Federal do Rio Grande do Sul - UFRGS \\ tania.koltermann@ufrgs.br
}

\begin{abstract}
Resumo: As emoções estão presentes constantemente no dia-a-dia das pessoas, no impacto que algumas situações causam, nas decisões que precisam ser tomadas e também nos processos de aprendizagem. Neste contexto, uma experiência emocional positiva pode contribuir para melhorar os processos cognitivos e comportamentais como a memória, a resolução de problemas e a criatividade, motivando o aluno e melhorando a sua eficiência no desenvolvimento de tarefas. De modo a produzir materiais de aprendizagem que atendam às necessidades emocionais dos alunos, existe a Engenharia Kansei, uma metodologia de desenvolvimento de produtos que traduz as emoções do usuário em especificações de projeto. Portanto, o objetivo deste artigo é a apresentação desta metodologia visando a produção de materiais de aprendizagem.
\end{abstract}

Palavras-chave: Emoção, Design instrucional, Engenharia Kansei, materiais de aprendizagem

The emotions are present constantly in the day-to-day lives, in the impact that certain situations cause, in the decisions that need to be taken and also in learning processes. In this context, a positive emotional experience may help improve cognitive and behavioral processes such as memory, problem solving and creativity, motivating students and enhancing their effectiveness in developing tasks. To produce learning materials that meet the emotional needs of students, there is the Kansei Engineering, a methodology for developing products that translates the emotions of the user at design specifications. Therefore, the aim of this article is the presentation of this methodology to the production of learning materials.

Keywords: Design, emotion, Kansei Engineering, learning materials

\section{INTRODUÇÃO}


As emoções geralmente foram tratadas desde os filósofos como Platão, Descartes e Kant de forma antagônica à cognição. Seguindo este viés, na Psicologia, por muito tempo, os estudos foram conduzidos tratando os processos cognitivos de maneira separada dos afetivos (DURAN, VENANCIO e RIBEIRO, 2004).

Em 1962, o psicólogo Piaget, em sua obra, A relação da afetividade com a inteligência no desenvolvimento mental da criança, afirma que o afeto desempenha um papel essencial no funcionamento da inteligência. Segundo ele, não há atos de inteligência sem que haja interesse no ponto de partida e regulação afetiva durante todo o curso de uma ação (PIAGET, 1962). Foliot e Michel (1998) também trabalham a ideia de que a emoção é um dos componentes fundamentais do processo cognitivo. Outros estudos também mostram esta perspectiva, como o de Duran, Venancio e Ribeiro (2004) e o de Ochsner e Phelps (2007). Isen e Reeve (2005) constataram que um estado emocional positivo pode promover a motivação, o comportamento de trabalho responsável, permitindo completar com sucesso e de forma mais eficiente uma tarefa.

No contexto de desenvolvimento de materiais de aprendizagem, como as emoções dos alunos podem ser consideradas? Neste sentido, a Engenharia Kansei pode ser empregada para a tradução dos sentimentos dos alunos em especificações de projeto. Para utilizá-la é importante o conhecimento sobre como funcionam as emoções e os estados emocionais, tema que será abordado a seguir.

\section{AS EMOÇÕES}

Apresentam-se na literatura diversos conceitos sobre o que se constitui a emoção, assim como diferentes maneiras de pensar sobre como esta ocorre e quais são os componentes que a integram.

Para Scherer (1984), a emoção consiste em um processo dinâmico em que é avaliada a relevância de um estímulo a partir das necessidades e preferências do indivíduo. Após, o organismo do indivíduo prepara-se para as ações fisiologicamente, apresentando reações ao estímulo de volta ao ambiente como as expressões corporais e faciais, por exemplo. Ao final, o indivíduo faz uma reflexão sobre o estímulo ocorrido e o armazena em sua memória.

As emoções teriam como função a constante avaliação de estímulos externos (do ambiente) ou internos (do próprio indivíduo, através do pensamento ou da memória) conforme a sua relevância e a preparação das reações comportamentais como resposta a estes estímulos. Portanto, Scherer (1984) considera a emoção como uma interface entre o organismo e o ambiente, que faz as mediações entre as constantes mudanças de situações e eventos $e$ as respostas individuais de comportamento.

Além do entendimento do que são as emoções, outra questão importante no desenvolvimento de produtos com foco emocional é a compreensão dos estados emocionais.

\subsubsection{Descrição dos estados emocionais}

Por mais de meio século, pesquisadores que atuam na área das emoções tentam estabelecer formas para representar concisamente um estado emocional após o recebimento de um estímulo. A solução encontrada foi a utilização de um Espaço Dimensional. Estes pesquisadores, através de diversos estudos estatísticos, apontaram 
as principais dimensões que auxiliam na representação de um estado emocional. Estas dimensões são eixos apresentando polos opostos que permitem a localização dentro deste espaço, representando de forma satisfatória similaridades e diferenças no significado dos estados afetivos (FONTAINE et al, 2007).

Russel (1980), por exemplo, propõe duas dimensões principais que são relacionadas à avaliação e à intensidade, resultando no Modelo Circumplexo do Afeto (Figura 1), que é baseado na visão do conceito de afeto por pessoas leigas e também a partir de análises multivariadas de estados afetivos autorrelatados.

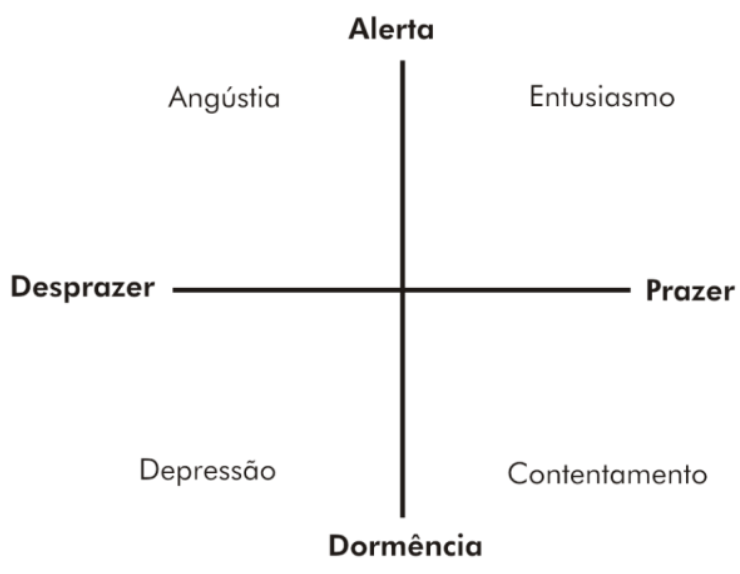

Figura 1 - Modelo Circumplexo do Afeto

Fonte: traduzido de Russel (1980)

No modelo de Russel (1980), as dimensões principais prazer-desprazer e alertadormência foram dispostas como eixos principais. As outras emoções como o entusiasmo, o contentamento, a depressão e a angústia seriam combinações entre as dimensões principais. Portanto, ao receber um estímulo, ocorre a avaliação deste no que se refere ao prazer ou o seu oposto, o desprazer. Além disso, verifica-se qual é a intensidade deste sentimento (RUSSEL e MEHRABIAN, 1977; RUSSEL, 1980).

Os três autores Osgood, Suci e Tannenbaum (1957) identificaram três dimensões (Avaliação, Potência e Atividade) que são dominantes nos julgamentos que realizamos de algum estímulo, sendo esta última ligada ao controle do ambiente. Ao propor o modelo tridimensional das emoções, estes autores também desenvolveram uma técnica de autorrelato chamada de Diferencial Semântico, que permite que uma pessoa avalie seu estado emocional, sendo apresentada a seguir.

\subsection{Técnica do Diferencial Semântico}

De acordo com Mehrabian e Russel (1977), existem formas de mensuração para cada uma das dimensões principais da emoção. O estado de prazer pode ser acessado através de autorrelato, utilizando a técnica do Diferencial Semântico de Osgood, Suci e Tannenbaum (1957), e através de indicadores de comportamento, expressões faciais e posturais. Já o grau de intensidade pode ser diretamente avaliado por relatório verbal ou por indicadores de comportamento como atividade vocal, atividade facial, velocidade de fala e volume da voz, podendo ser mensurado também pela técnica do Diferencial Semântico. E a Atividade, no âmbito de comportamento, pode ser medida em termos de postura corporal, mas também pode ser acessada a 
partir de relatórios verbais e pelo uso do Diferencial Semântico (YANI-DE-SORIANO e FOXALL, 2006).

De acordo com Osgood, Suci e Tannenbaum (1957), testes de mensuração fisiológica, como aqueles que examinam a reação salivar ou que medem a condução elétrica da pele, não apresentavam resultados satisfatórios e necessitavam de equipamentos caros e complexos para efetuá-los. Com a produção de resultados que os autores consideravam duvidosos, seria necessária a criação de uma forma alternativa de mensuração: o Diferencial Semântico.

A técnica constitui-se na aplicação de um instrumento composto por escalas de pares de adjetivos que são opostos. O respondente faz um julgamento de algum estímulo ou conceito (Pai), marcando um " $x$ " em cada escala (Figura 2). Quanto mais a sua marcação está localizada perto dos polos, mais o seu julgamento está próximo do adjetivo apresentado; marcando-se um " $x$ " no meio da escala, representa-se a neutralidade ou a irrelevância dos significados relacionados ao contexto (OSGOOD, SUCI e TANNENBAUM, 1957).

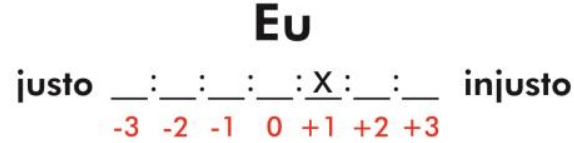

$$
\begin{aligned}
& \text { EU } \\
& \text { justo } \frac{-}{1}: \frac{-}{2}: \frac{-}{4}: \frac{x}{5}: \frac{-}{6}: \frac{-}{7} \text { injusto }
\end{aligned}
$$

Figura 2 - Escala com sete gradações

Fonte: traduzido de Osgood, Suci e Tannenbaum (1957)

Os dados brutos do Diferencial Semântico consistem em uma coleção de marcações nas escalas bipolares. A cada grau é atribuído um número: 1, 2, 3, 4, 5, 6 e 7 ou $-3,-2,-1,0,+1,+2,+3$. Esta última forma representa melhor a natureza bipolar das escalas (OSGOOD, SUCI e TANNENBAUM, 1957).

O Diferencial Semântico também é empregado pela Engenharia Kansei, que é uma metodologia que traduz as emoções em requisitos de produto (NAGAMACHI, 2011), que será apresentada a seguir.

\subsection{Engenharia Kansei}

A Engenharia Kansei (EK) também é conhecida por Engenharia Afetiva ou Engenharia Emocional. O termo japonês Kansei tem uma interpretação ampla abrangendo a cognição, tato, visão, olfato, cheiro, paladar assim como desejo, necessidade, sentimento, sensação, afeto, emoção e assim por diante. Segundo Nagamachi (2011), o Kansei é o resultado através da cognição e dos 5 sentidos; este autor afirma que a cognição seria o sexto sentido. Schütte (2005), no contexto da Psicologia, afirma que a palavra Kansei está relacionada a conceitos como sensação, percepção e cognição. O Kansei não seria apenas um processo que ocorre internamente, mas que está em constante recebimento de estímulos do ambiente, sendo necessário reconhecê-los e processá-los internamente e refletindo externamente o que foi processado. 
A Engenharia Kansei começou a ser estudada por Nagamachi em 1970. O autor da metodologia afirma que já foram desenvolvidos mais de 40 produtos, não apenas no Japão mas também em outras partes do mundo. Os produtos mais conhecidos são o carro Miata (MX-5), desenvolvido pela empresa Mazda em 1987, e o visor de cristal líquido (LCD) para câmeras da empresa Sharp, criado em 1980, recebendo o Good Design Award, do governo japonês (NAGAMACHI, 2011).

Desde a sua criação, inúmeras publicações foram dedicadas ao assunto como os artigos científicos, publicados de forma pulverizada em outras obras. Yang (2009) constata que na década de 90, os estudos sobre a Engenharia Kansei multiplicaram-se pela Ásia. Em 1998, surge a Japan Society of Kansei Engineering (JSKE), sediada em Tóquio, disseminando ainda mais os conhecimentos sobre a metodologia afetiva (NAGAMACHI, 2011).

Em relação à literatura brasileira, de acordo com El Marghani et al (2011), encontram-se poucas publicações sobre a Engenharia Kansei; e as existentes apresentam conteúdos muito simplificados sobre o assunto em relação a publicações de outros países, onde aplicam a Engenharia Kansei de modo extensivo, detalhando-a em sua literatura. Shimizu et al (2004) acreditam que a Engenharia Kansei pode ser aplicada em uma diversidade de áreas como o desenvolvimento de produtos físicos (projeto, fabricação e varejo) e de softwares (interfaces, educação e banco de dados), assim como, para o planejamento e controle urbano. Schütte (2005) e Nagamachi e Lokman (2011) afirmam que a Engenharia Kansei pode ser empregada tanto para o desenvolvimento de novos produtos quanto para o aperfeiçoamento dos produtos já existentes, ou até mesmo para aprimorar determinados elementos de um produto.

De acordo com Nagamachi e Lokman (2011), a Engenharia Kansei constituise em uma metodologia que traduz os sentimentos do consumidor em especificações de projeto para o desenvolvimento de um produto, sendo baseada em disciplinas como Matemática, Estatística, Psicologia, Marketing e Engenharia. Esta metodologia pode ser utilizada em qualquer momento em que seja necessário tomar decisões a respeito de aspectos de design.

No entanto, segundo Schütte (2002 e 2005), a falta de transparência da metodologia em si e dos procedimentos para o desenvolvimento de produto utilizando a Engenharia Kansei, dado a sua pesada parte estatística, foi um dos motivos para a lenta disseminação desta metodologia, quando comparado ao QFD. Enquanto os procedimentos desta técnica mostram-se mais fáceis de serem compreendidos, na Engenharia Kansei, necessita-se de conhecimentos aprofundados na área da Matemática e Estatística. O autor desenvolveu um modelo simplificado conforme mostra a Figura 3: 


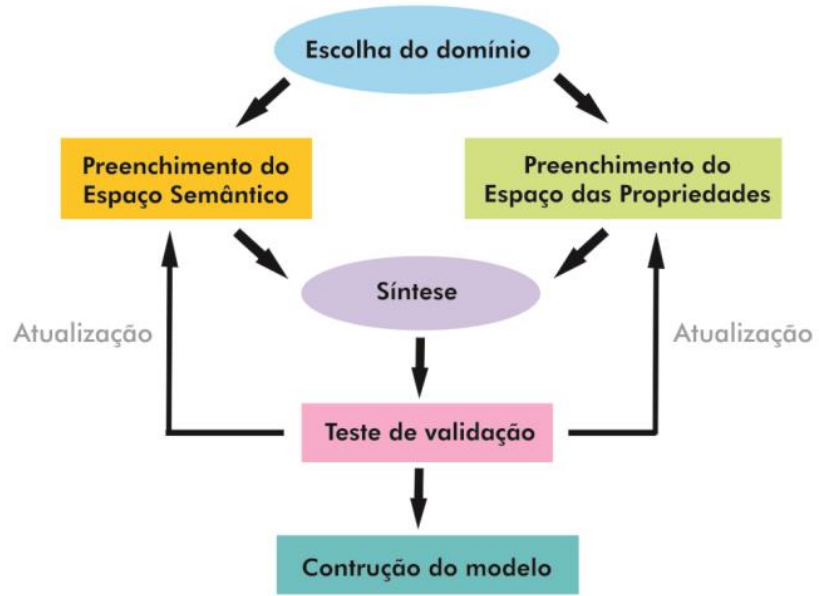

Figura 3 - Modelo simplificado da Engenharia Kansei

Fonte: traduzido de Schütte (2005)

O processo de desenvolvimento de produto que utiliza a Engenharia Kansei inicia com a escolha de um domínio. Esta fase tem a função de construir o conceito ideal do produto. Após o produto ser descrito a partir de uma ideia, as etapas seguintes constituem-se no preenchimento do Espaço Semântico e do Espaço das Propriedades.

O Espaço Semântico é composto por palavras Kansei que descrevem o domínio do produto. Estas palavras podem ser coletadas a partir de diversas fontes como revistas, manuais, literatura, especialistas, usuários experientes etc. Geralmente são adjetivos, mas se aceita também verbos e substantivos (NAGAMACHI, 2011). Nagamachi e Lokman (2011) sugerem a coleta de 300 a 800 palavras para compor o Espaço Semântico. De acordo com Schütte (2005), o Espaço das Propriedades, é composto por propriedades ou atributos de um produto que são características que podem ser avaliadas. Com este mesmo objetivo, utiliza-se também o termo elemento de design. Como exemplos de propriedades de produtos, podem ser citados: cor, tipografia, tamanho, velocidade, altura, largura, etc. Através da Engenharia Kansei investigam-se quais destes elementos estão relacionados com as emoções de quem utiliza um produto (LOKMAN, 2009). Estas duas descrições (semântica e das propriedades do produto) constituem-se uma espécie de espaço vetorial que são analisados de forma relacionada na fase de Síntese, indicando quais propriedades podem provocar algum impacto emocional em quem utiliza o produto (SCHÜTTE, 2002 e 2005).

A próxima fase da Engenharia Kansei consiste na validação dos dados encontrados, que dependerá dos métodos empregados anteriormente. Na última fase, que é opcional, poderá ser construído um modelo matemático ou não que dependerá do método utilizado na fase de Síntese para obter o conceito do produto a ser desenvolvido (SCHÜTTE, 2002 e 2005).

Embora, o Kansei seja uma impressão individual a partir de um determinado estímulo, é possível investigar quais são as percepções de um grupo de pessoas relacionadas a um produto ou a alguma característica deste utilizando a Engenharia 
Kansei. De acordo com Lokman (2009), um produto Kansei é um produto inteligente no qual se tenta prever as respostas emocionais do usuário no seu processo de desenvolvimento. Segundo esta autora, um processo de desenvolvimento de produto baseado em concepções erradas sobre os desejos dos usuários pode resultar em um fracasso comercial, sendo a Engenharia Kansei uma alternativa para o reconhecimento e tradução destas necessidades, inserindo-as neste processo, minimizando a possibilidade de fracasso comercial do produto.

No caso desta pesquisa, a Engenharia Kansei pode ser utilizada para captar o Kansei dos alunos em relação a um material de aprendizagem de natureza audiovisual. A Engenharia Emocional pode auxiliar no conhecimento de quais elementos de design de um material de aprendizagen provocam determinadas emoções.

Devido à diversidade de aplicações, existem pelo menos três tipos de Engenharia Kansei. É necessário o conhecimento sobre estes tipos para que seja possível selecionar quais são as ferramentas e métodos a serem utilizados. Estes três tipos serão apresentados a seguir.

\subsubsection{Tipos de Engenharia Kansei}

A Engenharia Kansei Tipo I, entre os três tipos, é a mais fácil de compreender e introduzir. Constitui-se na técnica fundamental do método da Engenharia Kansei. 0 Kansei do usuário é relacionado manualmente (sem o auxílio de um software). propriedades do produto para servir de orientação no desenvolvimento de produtos. A Figura 4 mostra a visão geral do Tipo I com a indicação das macrofases de Schütte (NAGAMACHI, 1995; NAGAMACHI e LOKMAN, 2011; SCHÜTTE, 2005).

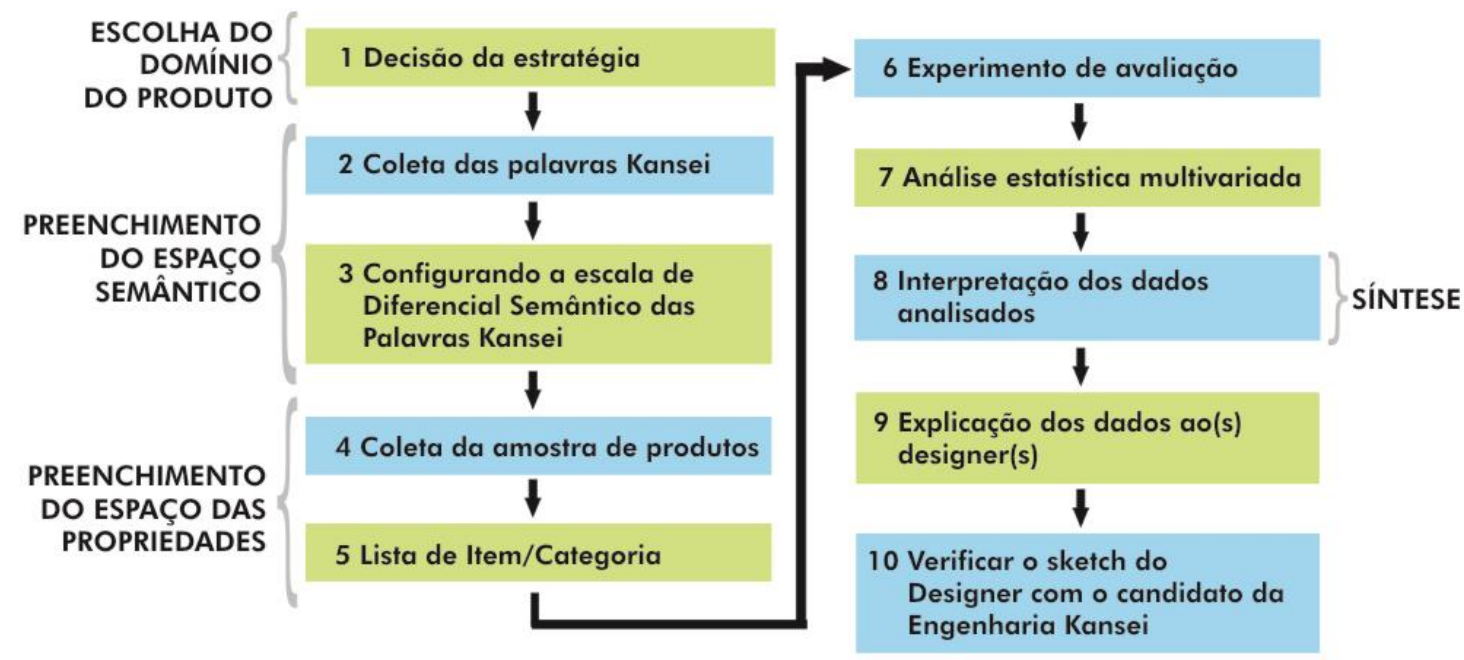

Figura 4 - Processo da Engenharia Kansei Tipo I

Fonte: traduzido e adaptado de Schütte (2005) e Nagamachi e Lokman (2011)

O processo do Tipo I inicia com a Decisão da Estratégia (1). Nesta fase, o presidente, os gerentes e os designers definem as estratégias de desenvolvimento do novo produto. $O$ ponto principal é compreender quais são as exigências da empresa para a produção do produto. A fase 2 refere-se à coleta de palavras Kansei 
relacionadas com o domínio do produto. Estas são coletadas a partir da literatura e/ou relatos de usuários e especialistas. Nesta fase, é importante coletar o maior número de palavras possíveis, podendo alcançar 800 palavras, compondo o Espaço Semântico do produto. A partir destas palavras, extraem-se quais são as palavras-chave que definem o produto, e este procedimento pode ser feito de duas maneiras.

As palavras-chave são importantes, pois na fase 3 , escalas de Diferencial Semântico são criadas a partir destas para serem futuramente aplicadas junto ao público a qual se destina o produto. Posteriormente, tem-se a fase 4 , que consiste na coleta de produtos que são similares ou que tenham alguma característica semelhante ao produto que será criado. A partir desta amostra é feita uma lista de itens e categorias a partir das propriedades encontradas (fase 5). Na sexta fase, é aplicado o conjunto de escalas de Diferencial Semântico (anteriormente desenvolvida na fase 3) com produtos similares coletados ao público que utiliza o produto.

Com os resultados, faz-se uma análise estatística multivariada (7) utilizando diversas técnicas e ferramentas. Na fase 8, ocorre a interpretação dos dados, sendo possível relacionar os estados afetivos com os elementos de design. Na próxima fase, os dados são apresentados e explicados ao(s) designer(s) ou à equipe de projeto, sendo que este(s) na última fase $(10)$ avalia(m) se deve $(\mathrm{m})$ usar ou não os elementos de design e qual sua forma de uso no desenvolvimento do novo produto (NAGAMACHI, 2011; NAGAMACHI e LOKMAN, 2011).

O Tipo I, mesmo sendo mais simples de compreender e aplicar entre os três tipos de Engenharia Kansei, pode ser utilizado no desenvolvimento de produtos complexos. Por exemplo, o carro Miata (MX-5) da empresa Mazda, que obteve um grande sucesso comercial, foi desenvolvido com a utilização deste tipo de Engenharia Kansei (NAGAMACHI, 2011; NAGAMACHI e LOKMAN, 2011).

Assim como o Tipo I, o processo da Engenharia Kansei Tipo II (Sistema da Engenharia Kansei), é iniciado a partir das necessidades emocionais dos consumidores e a partir do conceito do produto. No entanto, para converter as emoções em requisitos de projeto, inclui o uso de um banco de dados e de programas de computador que utilizam técnicas como: Sistema Especialista, Rede Neural, Algoritmo Genético, Lógica Fuzzy, etc. A Figura 5 mostra a metodologia do processo (NAGAMACHI, 2011; NAGAMACHI e LOKMAN, 2011).

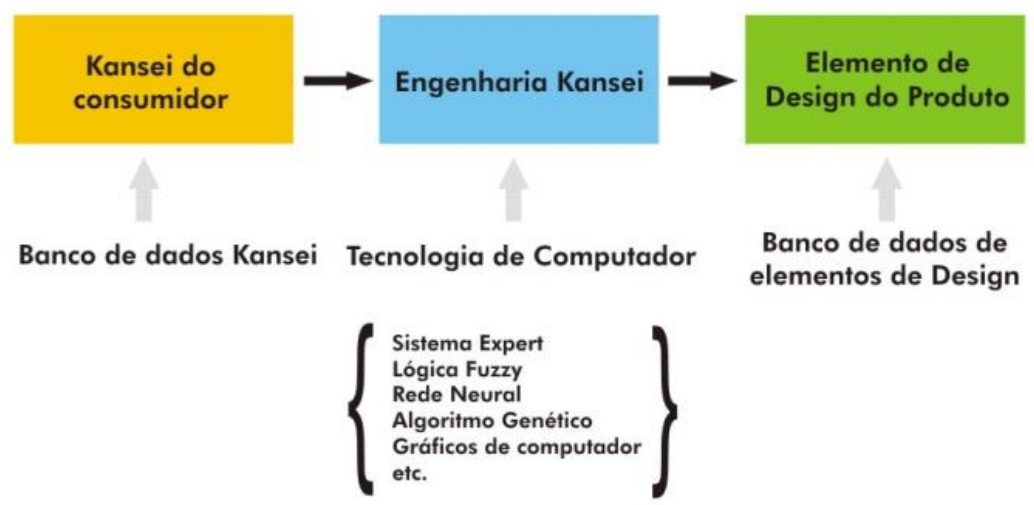

Figura 5 - Processo Engenharia Kansei Tipo II

Fonte: traduzido de Nagamachi e Lokman (2011) 
Uma das aplicações do Tipo II é o HULIS (acrônimo de Human Living System), um sistema que auxilia no processo de decisão do consumidor. Primeiramente, o consumidor coloca as palavras que representam o Kansei desejado em relação à casa a ser construída. Neste exemplo, o banco de dados de palavras Kansei é composto por termos recorrentes na indústria da construção e identifica a estrutura das palavras através de Análise Fatorial Em relação aos elementos de design, estes são desmembrados em várias especificações em banco de dados. O sistema apresenta perguntas relacionando os elementos com as palavras Kansei. Ao respondê-las, o consumidor indica quais são os requisitos de projeto que irão satisfazer o seu Kansei (NAGAMACHI, 2011; NAGAMACHI e LOKMAN, 2011).

Na Engenharia Kansei Tipo III (Modelagem da Engenharia Kansei), utiliza-se um modelo matemático para fazer a mediação, sendo as relações das propriedades físicas com as emoções estabelecidas através de um coeficiente de valor. A aplicação desta Engenharia Kansei Tipo III foi feita no desenvolvimento de uma impressora da empresa japonesa Sanyo, que permitia a troca da sua cor original (NAGAMACHI, 2011; NAGAMACHI e LOKMAN, 2011).

Conhecida a Engenharia Kansei, seus tipos e o seu funcionamento, parte-se para a sua aplicabilidade no contexto educacional.

\subsection{A Engenharia Kansei no contexto educacional}

Para Chuah, Chen e Teh (2008) a Engenharia Kansei se constitui uma ferramenta adicional para auxiliar nos momentos em que o designer instrucional necessita tomar decisões relacionadas aos elementos de design de um material de aprendizagem. Segundo estes autores, a Engenharia Kansei pode ser utilizada para descobrir quais são os elementos de design que podem ser empregados para promover emoções positivas, otimizando os efeitos da aprendizagem. Os autores propõem algumas adaptações. Com relação à seleção das palavras Kansei, o designer instrucional deve também colher adjetivos relacionados com o processo de aprendizagem.

Chuah, Chen e Teh (2008) acreditam que a seleção de propriedades que normalmente ocorre analisando produtos similares disponíveis no mercado, no caso de projetos de Design Instrucional, não funciona da mesma maneira. Como experimento, estes autores apontam que devem ser desenvolvidos vários materiais educacionais: o primeiro seria formado por todos os elementos de design instrucionais; o segundo, com todos, exceto com os objetivos de aprendizagem; o terceiro, com todos, exceto, com o sequenciamento de conteúdo, e assim por diante. O aluno faria a avaliação de todos os materiais através de questionário com escalas de Diferencial Semântico. A avaliação de um aluno em relação a um elemento de design específico pode ser feita utilizando várias técnicas, embora, a forma mais utilizada seria a partir de escalas de palavras no que se refere ao julgamento do que é bom ou ruim.

No entanto, a experiência de se seguir a metodologia da Engenharia Kansei no que tange a utilização de produtos similares também continua válida, como propõe Nagamachi (2005), pois nem sempre se dispõe de uma equipe de desenvolvimento, de recursos financeiros e tempo hábil de produção para realizar a sugestão de Chuah, Chen e Teh (2008). Uma experiência na produção de materiais de aprendizagem audiovisuais voltados para a TV Digital foi realizada em 2012 utilizando a Engenharia 
Kansei seguindo a metodologia do Tipo I. Contrapondo a ideia de Chuah, Chen e Teh (2008), esta pesquisa utilizou para a seleção de propriedades, produtos audiovisuais similares, já que era a primeira vez que estava sendo desenvolvidos estes conteúdos para a TV Digital e era necessário entender quais elementos de design estariam ligados a estados emocionais positivos dos alunos (SUGIMOTO, 2013).

Portanto, estas sugestões de adaptações na Engenharia Kansei para o contexto educacional são importantes, pois auxiliam no aprimoramento da metodologia para este foco.

\section{CONCLUSÃO}

Este estudo também contribuiu para o desenvolvimento do campo teórico que relaciona o Design e as emoções, que tem crescido nos últimos anos e também para aumentar a literatura existente sobre esta metodologia no Brasil, já que existem poucas publicações científicas no país (SUGIMOTO, SILVA e VAN DER LINDEN, 2012; EL MARGHANI et al, 2011). Além disso, constatou-se a aplicação da Engenharia Kansei no desenvolvimento de vários produtos como automóveis, câmeras filmadoras, cosméticos, artigos de vestuário, tratores e websites, como apresentam Nagamachi (2011), Schütte (2002) e Lokman (2009), no entanto, existe a necessidade de desenvolvimento de pesquisas relacionadas à aplicação em materiais de aprendizagem, de forma a atender cada vez mais satisfazer os alunos.

Além disso, a literatura sobre a metodologia da Engenharia Kansei nem sempre é de fácil compreensão devido aos conhecimentos necessários sobre os métodos estatísticos utilizados e também devido à falta de publicações em português. Portanto, é de fundamental importância haja publicações sobre o tema já que a metodologia envolve a combinação de conhecimentos de várias áreas como a Matemática, a Estatística, a Psicologia, o Design e a Engenharia, entre outros, o que torna a sua compreensão complexa (SCHÜTTE, 2002).

Existem, inclusive, adaptações como as Chuah, Chen e Teh (2008) para facilitar a aplicação da metodologia no desenvolvimento de produtos. Cabe a equipe de desenvolvimento a verificação da aplicabilidade da Engenharia Kansei em seu projeto e das adaptações necessárias.

Ao considerar a utilização a Engenharia Kansei em projetos de desenvolvimento de produtos, a equipe deve ter conhecimentos sobre Estatística ou ter um assessoramento de um profissional da área. O domínio sobre as técnicas estatísticas, sobre os softwares e complementos que realizam estas análises é muito importante e requer conhecimentos específicos, assim como, a interpretação dos dados apresentados pelos softwares. Quando for do desejo da equipe de desenvolvimento utilizar a Engenharia Kansei Tipos II e III, será necessário também o auxílio de um programador.

Portanto, assim como afirma El Marghani et al (2011), a Engenharia Kansei constitui-se em uma metodologia de desenvolvimento de produto muito importante, podendo contribuir nas decisões de projeto, pois utiliza informações mais precisas provenientes de dados que originaram dos usuários.

\section{REFERÊNCIAS}


CAUDURO, Flávio Vinícius. Design gráfico \& pós-modernidade. Revista FAMECOS. Porto Alegre, n. 13, dez. 2000. Disponível na internet por http em: $<$ http://revistaseletronicas.pucrs.br/ojs/index.php/revistafamecos/article/viewFile/30 88/2364>. Acesso em: 22 out. 2012.

CHUAH, Kee Man; CHEN, Chwen Jen; TEH, Chee Siong. Kansei Engineering Concept in Instructional Design. A Novel Perspective in Guiding the Design of Instructional Materials. In: INTERNATIONAL CYBERSPACE CONFERENCE OF ERGONOMICS, 5., 2008, Kuching. Anais... Disponível na internet por http em: <http://unimas.academia.ed u/kmchuah/Papers/856146/Kansei Engineering Concept in Instructional Design A Novel Perspective in Guiding the Design of Instructional Materials>. Acesso em: 10 jul. 2012.

DURAN, Kelly Marion; VENANCIO, Kelly Marion; RIBEIRO, Lucas dos Santos. Influência das Emoções na Cognição, 2004. Disponível na internet por http em: <http://www.ic.unicamp.br/ wainer/cursos/906/trabalhos/Trabalho E1.pdf>. Acesso em: 13 fev. 2011.

EL MARGHANI, Viviane G. R.; GABARDO, Ana Claudia; SILVA, Alex Sandro de A.; SILVA, Felipe C. da; SALATA, Natalia S. Kansei Engineering: metodologia orientada ao consumidor para suporte a decisão de projeto. In: CONGRESSO BRASILEIRO DE GESTÃO DE DESENVOLVIMENTO DE PRODUTO, 8., 2011, Porto Alegre. Anais... Disponível na internet por http em: <http://www.ufrgs.br/cbgdp2011/downloads/8954.pdf>. Acesso em: 18 ago. 2012.

FOLIOT, Gérald; MICHEL, Olivier. Learning Object Significance with an Emotion based Process, 1998. Disponível na internet por http em: < $\underline{\text { http://alphaworks.ishlyo }}$ n.cnrs.fr/Documents/PDF/foliot.sab.pdf>. Acesso em: 13 fev. 2011.

FONTAINE, Johnny R. J.; SCHERER, Klaus R.; ROESCH, Etienne B.; ELLSWORTH, Phoebe C. The world of emotions is not Two-Dimensional. Psychological Science, v.18, n.22, 2007.

ISEN, Alice M.; REEVE, Johnmarshall. The Influence of Positive Affect on Intrinsic and Extrinsic Motivation: Facilitating Enjoyment of Play, Responsible Work Behavior, and Self-Control. Motivation, v. 29, n.4, dez. 2005.

LOKMAN, Anitawati Mohd. Emotional User Experience in Web Design: The Kansei Engineering. 2009. Tese (Doutorado em Filosofia) - Faculty of Computer and Mathematical Sciences, Universiti Teknologi Mara, Selangor Darul Ehsan, 2009. Disponível na internet por http em: <http://anitawati.uitm.edu.my/Pub.html>. Acesso em: 4 jun. 2012.

NAGAMACHI, Mitsuo. Kansei Engineering: A new ergonomic consumer-oriented technology for product development. International Journal of Industrial Ergonomics, v.15, p. 3-11, 1995.

Kansei/Affective Engineering and History of Kansei/Affective Engineering in the World. In: NAGAMACHI, Mitsuo (Ed.). Kansei/Affective Engineering. Boca Raton: CRC Press, 2011.

NAGAMACHI, Mitsuo; LOKMAN, Anitawati Mohd. Innovations of Kansei Engineering. Boca Raton: CRC Press, 2011. 
OCHSNER, Kevin N.; PHELPS, Elizabeth. Emerging perspectives on emotion-cognition interactions. TRENDS in Cognitive Sciences. Nova lorque, v. 11, n. 8. p. 317-318, jul. 2007. Disponível na internet por http em: <http://www.psych.nyu.edu/phelpslab/pa pers/07 TCS V11No8.pdf>. Acesso em: 13 fev. 2011.

OSGOOD, Charles E.; SUCl, George J.; TANNENBAUM, Percy H. The Measure of Meaning. Urbana: University of Illinois Press, 1957.

PIAGET, Jean. A relação da afetividade com a inteligência no desenvolvimento mental da criança. Tradução de Magsa Schu. Disponível na internet por http em: <http://www.marcelo.kinghost.net/ufrgs/CINTED/louise\%20e\%20marcia/aula3/piaget -afetividade 1 .pdf >. Acesso em: 29 jan. 2012.

RUSSEL, James A. A Circumplex Model of Affect. Journal of Personality and Social Psychology. Washington, v.39, n.6, p.1161-1178, 1980.

RUSSEL, James A.; MEHRABIAN, Albert. Evidence for a three-factor Theory of Emotions. Journal of Research in Personality, v.11, p. 273-294, 1977.

SCHERER, Klaus R. On the nature and function of Emotion: A Component Process Approach. In: SCHERER, Klaus R.; EKMAN, Paul (Org.). Approaches to Emotion. Hillsdale: Lawrence Erlbaum, 1984.

SCHÜTTE, Simon. Designing Feelings into Products: Integrating Kansei Engineering Methodology in Product Development. 2002. 115f. Tese (Doutorado em Ciência e Tecnologia) - Department of Management and Engineering, Machine Design, Linköping University, Linköping, 2002. Disponível na internet por http em: $<$ http://liu.divaportal.org/smash/record.jsf?pid=diva2:19998>. Acesso em: 10 jul. 2012.

Engineering Emotional Values in Product Design: Kansei Engineering in Development. 2005. 106f. Dissertação (Mestrado em Ciência e Tecnologia) Department of Management and Engineering, Machine Design, Linköping University, Linköping, 2005.

SHIMIZU, Yoshio; TSUGUTAKE, Sadoyama; KAMIJO, Masayoshi; HOSOYA, Satoshi; HASHIMOTO, Minoru; OTANI, Tsuyoshi; YOKOI, Kouich; HORIBA, Yousuke; TAKATERA, Masayuki; HONYWOOD, Michael, INUI, Shigeru. On-demand production system of apparel on the basis of engineering. International Journal of Clothing Science and Technology, v.16, n.1, p.32-42, 2004.

SUGIMOTO, Adriana. Requisitos de projeto para o desenvolvimento de materiais de aprendizagem para a TV Digital a partir da aplicação da Engenharia Kansei. 2013. Dissertação (Mestrado em Design) - Programa de Pós-Graduação em Design, Universidade Federal do Rio Grande do Sul, Porto Alegre, 2013. Disponível na internet por http em: <http://hdl.handle.net/10183/79835>. Acesso em: 10 maio 2014.

SUGIMOTO, Adriana; SILVA, Tânia Luisa Koltermann da; VAN DER LINDEN, Júlio Carlos de Souza. A study on Design and Emotion Books from the 1980 to 2011. In: INTERNATIONAL CONFERENCE ON DESIGN \& EMOTION, 8, 2012, Londres. Anais... BRASSETT, Jamie; HEKKERT, Paul; LUDDEN, Geke; MALPASS, Matt, MCDONELL, Janet (Ed.). Out of control: Book of Abstracts. London: University of Arts London, 2012. 
TIMM, Maria Isabel; BOS-MIKICH, Adriana; THADDEU, Roberto; AZEVEDO, Ana Maria P.; CABRAL JÚNIOR, Paulo Augusto. Vídeos agregam valor ao trabalho do professor de ciências da saúde. Revista Renote, v. 6, n. 2, dez. 2008. Disponível na internet por http em: <http://seer.ufrgs.br/renote/article/view/14679>. Acesso em: 29 nov. 2012.

YANG, Chih-Chieh. A Study on Variable Selection for Kansei Engineering System: Applications for Product Design. Berlim: VDM Verlag Dr. Müller, 2009.

YANI-DE-SORIANO, Mirella; FOXALL, Gordon R. The emotional power of place: The fall and rise of dominance in retail research. Journal of Retailing and Consumer Services, v. 13, p. 403-416, 2006. 